\title{
Energy Balanced Redeployment Algorithm for Heterogeneous Wireless Sensor Networks
}

\author{
Guang Ye, Baihai Zhang, Senchun Chai, and Lingguo Cui \\ School of Automation, Beijing Institute of Technology, Beijing 100081, China \\ Correspondence should be addressed to Senchun Chai; chaisc97@bit.edu.cn
}

Received 15 August 2014; Accepted 1 September 2014

Academic Editor: Yun-Bo Zhao

Copyright (C) 2015 Guang Ye et al. This is an open access article distributed under the Creative Commons Attribution License, which permits unrestricted use, distribution, and reproduction in any medium, provided the original work is properly cited.

\begin{abstract}
Wireless sensor networks (WSNs) have gained worldwide attention in recent years. Since WSNs can be conveniently deployed to monitor a given field of interest, they have been considered as a great long-term economic potential for military, environmental, and scientific applications and so forth. One of the most active areas of research in WSNs is the coverage which is one of the most essential functions to guarantee quality of service (QoS) in WSNs. However, less attention is paid on the heterogeneity of the node and the energy balance of the whole network during the redeployment process. In this work, the energy balanced problems in mobile heterogeneous WSNs redeployment have been analyzed. The virtual force algorithm with extended virtual force model is used to improve the QoS of the deployment. Furthermore energy model is added to enhance or limit the movement of the nodes so that the energy of nodes in the whole WSNs can be balanced and the lifetime of the networks can be prolonged. The simulation results verify the effectiveness of this proposed algorithm.
\end{abstract}

\section{Introduction}

WSNs consist of small-sized low cost sensor nodes which have several restrictions in energy supply, computing power, and bandwidth of the wireless communication [1]. The QoS of the network is highly related to the deployment performance of the nodes. However, in many working environments such as disaster areas, battlefields, and toxic gas regions, all sensor nodes are mainly deployed randomly by aircraft instead of human beings. In such case, the deployment result may not satisfy the requirement of the system. All the nodes may cluster in a small region or may even distribute sparsely without connectivity guarantee which would influence the QoS and reliability of the WSNs significantly.

Many researchers are currently engaged in coverage problem [2-4] and numerous algorithms are related to mobile WSNs.

Mobile WSNs are composed of a distributed collection of nodes, each of which has communication, sensing, computation, and locomotion capabilities. The mobility of sensor nodes allows more complex application scenarios. With locomotion capabilities, sensor nodes can adjust their positions after stochastic distribution; thus the whole networks could enhance the coverage performance and reach more precise placement. However, the movement of the node would expend the energy of the node. Some node may run out of power because of the long distance movement during redeployment.

Another actual problem in WSNs coverage is that the nodes in networks cannot be always the same in practice due to various reasons. The coverage analysis of heterogeneous WSNs is very useful for both academic and industrial fields. The concept of heterogeneous sensor network is proposed in [5]. The existence of heterogeneous WSNs is mainly due to the following aspects. (1) Nodes are different as physical devices, and the physical properties of the sensor node are very difficult to be completely homogenized. (2) The same type of sensor nodes may work differently due to work environment, regional terrain characteristics, and imbalanced workload or other reasons. All those could affect the behaviour of nodes in WSN.

In the literature, abundant work has been done in the redeployment of the mobile WSNs, and many effective algorithms have been put forward to obtain a required placement 
and improve the coverage rate. Pervious work on mobile WSNs redeployment algorithms can be classified into two main kinds: virtual force algorithms (VFA) and computational geometry-based approach. In VFA based algorithms [6], those models the mobile sensor nodes as electrons or molecules, and nodes move toward or away from each other by the virtual force (often related to the distance between nodes) or potential fields. However algorithms above do not consider some actuality problems such as connectivity maintenance and the energy cost [7]. References [8-10] are algorithms according to the computational geometry in which nodes update their positions to from a uniform Voronoi diagram or Delaunay triangulation. It can provide well performance but is hard to be used for the need of the global position information of the whole network which usually cannot be realized.

In this paper, the redeployment problem in mobile heterogeneous WSNs considering energy balanced is addressed. Delaunay triangulation is used in VFA to find the logic neighbors nodes in order to avoid nodes cluster in a small region. Furthermore according to the research fixed ideal distance is proposed to solve the serious coverage holes and redundant problem in traditional VFA. And the energy model of the nodes is proposed. In order to balance the energy of the whole networks, in this paper energy control function is added to the virtual force model.

Throughout the paper, it is assumed that the communication range is two times the sensing range. The rest of this paper is organized as follows. Section 2 gets over the past work that closely related to our work. Section 3 analyzes energy unbalanced problem in mobile WSNs redeployment and provides a solution. Section 4 gives the simulation results that illustrate the performance. Section 5 is the conclusion of the paper.

\section{Related Works}

The prior work on redeployment of mobile WSNs in recent years which closely related to this subject is summarized below.

The concept of redeployment of mobile WSNs is derived from dealing with coordination in behavior control of many robots teams [11, 12]. Gage [11] has classified the use of robot swarms to provide blanket, barrier, or sweep coverage of area. According to this classification, the redeployment problem studied in this paper focuses on the blanket coverage. Reference [12] considers multirobot exploration and mapping for larger teams and gives an evaluation of different strategies for coordinating the efforts of a robot team during an exploration mission in an unknown environment. In [13], a potential-field-based approach (PFBA) is presented to deploy sensor nodes in a target environment. In order to obtain a uniform deployment in an unknown enclosed area, control force defined as negative gradient of potential (NGOP) is employed. However, some crucial problems had not been considered such as connectivity maintenance and topology control. In [14], the author introduced the concept of virtual force algorithm (VFA) to the WSNs deployment.
The VFA adopts the similar strategy as PFBA, by considering the virtual attractive and repulsive forces exerted on each node by neighbor nodes and obstacles. The VFA can significantly increase sensor coverage. These works only consider homogeneous sensing models. Based on works in [14], a distributed self-spreading algorithm is developed in [15]. The virtual force in (distributed self-spreading algorithm) DSSA is modeled as internuclear repulsion and attraction between molecules. Computational geometry such as Voronoi diagram and Delaunay triangulation is commonly used in redeployment of WSNs, and the vector-based algorithm (VEC), the Voronoi-based algorithm (VOR), and MiniMax are presented in [8]. The algorithms above use Voronoi diagrams to divide the coverage field into many small areas and enhance the covered area by pushing or pulling nodes according to virtual force. Computational geometry-based algorithms can solve the stacking problem. However the Voronoi diagram is a global structure; it means that the diagram can be obtained only if the global location information of all nodes in the WSNs is known.

\section{Preliminaries}

3.1. Network Model and Assumptions. In this paper we focus on the redeployment problem of heterogeneous WSNs in 2dimensional plane. The position of node $i$ is described as $\left(x_{i}, y_{i}\right)$. The distance between node $i$ and node $j$ is defined as Euclidean distance $d_{i j}$. The initial deployment is a random deployment in unknown distribution. We assume that every node can learn its own position by GPS or other localization technologies. $R_{\mathrm{c}}$ is the communication range; if $d_{i j} \leq R_{\mathrm{c}}$, node $i$ and node $j$ are neighbor nodes. Nodes can receive and send message to their neighbors without losing data. Meanwhile, nodes can also get relative distances and orientations between them. Nodes are aware of their remaining energy and can share this information with their neighbors. $R_{\mathrm{s}}$ is the sensing range. The perceptual model $C_{p i}$ is defined as follows ( $p$ is an arbitrary position in target area):

$$
C_{p i} \begin{cases}1, & d_{i p}<R_{\mathrm{s}} \\ 0, & d_{i p} \geq R_{\mathrm{s}} .\end{cases}
$$

In general, $R_{\mathrm{c}}$ is larger than $R_{\mathrm{s}}$.

All of the virtual physics algorithms for redeployment problem in WSNs are similar to the structure of virtual force, which include the ideas of potential field with circle packing that models the sensor node to be a particle in the potential field. And the potential field exerts forces on the nodes in its field. For a couple of neighbor nodes $i$ and $j$, the potential function $V_{i j}$ could be built. And the virtual force is shown as follows:

$$
F_{i j}=-\nabla V_{i j}
$$

Nodes move toward the target area by the virtual forces. The force can be attractive force or repulsive force. Generally, there is an ideal distance $D_{t h}$. If the distance between nodes $i$ and $j$ is less than $D_{t h}$, repulsive force will act on nodes. Similarly, attractive force will act on nodes if the distance is 
larger than the ideal distance. The repulsive force is to insure the sensors are sparse enough without too much redundant area and the attractive force is to guarantee the coverage without coverage holes.

The control law for nodes is described in

$$
\ddot{\mathbf{x}}_{i}=\sum_{j \in G_{i}} \mathbf{F}_{i j}-k_{d} \dot{\mathbf{x}}_{i},
$$

where $\ddot{\mathbf{x}}_{i}$ is the acceleration of the node, $\dot{\mathbf{x}}_{i}$ is the velocity of the node, $k_{d}$ is damping coefficient, and $G_{i}$ is the neighbors of node $i$.

In traditional VFA, the virtual force is defined as follows:

$$
\mathbf{F}_{i j}= \begin{cases}\omega_{A}\left(d_{i j}-D_{t h(i, j)}\right) \mathbf{u}_{i j}, & \text { if } d_{i j}>D_{t h(i, j)} \\ \omega_{R} d_{i j}^{-1} \mathbf{u}_{j i}, & \text { if } d_{i j}<D_{t h(i, j)} \\ 0, & \text { if } d_{i j}=D_{t h(i, j)},\end{cases}
$$

where $\omega_{A}$ is the virtual force attractive coefficient, $\omega_{R}$ is the virtual force repulsive coefficient, $d_{i j}$ is the Euclidean distance between node $i$ and node $j$, and $\mathbf{u}_{i j}$ is the unit vector from node $i$ to node $j$.

Nodes move to new positions according to (3). The total force $F_{i}$ exerted on node $i$ can be described as follows:

$$
F_{i}=\sum_{j \in G_{i}} F_{i j}
$$

\section{Energy Balanced Redeployment Algorithm for Heterogeneous WSNs}

The extended virtual force algorithm is a redeployment algorithm with some novel features which can overcome the limitations of traditional VFA. In practical networks the sensing range $R_{\mathrm{s}}$ of nodes cannot be always the same due to various reasons; in that case coverage holes may appear which may influence the QoS of the WSNs seriously. Prior algorithm assumed that $R_{\mathrm{c}}$ is two times larger than $R_{\mathrm{s}}$; however as a matter of fact $R_{\mathrm{c}}$ is much larger than $R_{\mathrm{s}}$. High $R_{\mathrm{c}}$ may induce stacking problem in redeployment.

In order to solve the problem above, Energy Balanced Redeployment Algorithm (EBRA) is proposed.

Firstly we prove the stability of the algorithm.

4.1. Stability Analysis. The stability of the algorithm can be proved with Lyapunov stability theory.

Proof. Assume $x_{i}$ is the position vector of node $i$. The control input of node $i$ is the virtual force $F_{i j}$ exerted on node $i$ by its neighbor $j$. Then the virtual potential field can be known according to (2) and (5). The total force of the node $i$ can be summarized as follows:

$$
F_{i}=\sum_{j \in G_{i}} F_{i j}=\sum_{j \in G_{i}}-\nabla V_{i j} .
$$

Then energy function $\boldsymbol{\Phi}$ which combines kinetic energy with potential energy is built as Lyapunov function:

$$
\boldsymbol{\Phi}=\sum_{i=1}^{N} \frac{1}{2} \dot{x}_{i}^{T} \dot{x}_{i}+\frac{1}{2} \sum_{i=1}^{N} \sum_{j \in G_{i}} V_{i j} .
$$

For the symmetry of $V_{i j}$ and $V_{j i}, \dot{x}_{i}=-\dot{x}_{j}$ on the orientation from node $i$ to node $j$. The time derivative $V_{i j}$ is described as follows:

$$
\begin{aligned}
\frac{d}{d t} V_{i j} & =\frac{\partial V_{i j}}{\partial x_{i}} \cdot \frac{d x_{i}}{d t}+\frac{\partial V_{i j}}{\partial x_{j}} \cdot \frac{d x_{j}}{d t} \\
& =\nabla V_{i j} \cdot \dot{x}_{i}-\nabla V_{i j} \cdot \dot{x}_{i} \\
& =\nabla V_{i j} \cdot\left(\dot{x}_{i}-\dot{x}_{j}\right) \\
& =2 \nabla V_{i j} \cdot \dot{x}_{i} .
\end{aligned}
$$

The energy function $\Phi$ can be simplified as follows:

$$
\begin{aligned}
\dot{\boldsymbol{\Phi}} & =\sum_{i=1}^{N} \frac{1}{2}\left(\dot{x}_{i}^{T} \dot{x}_{i}+\dot{x}_{i}^{T} \dot{x}_{i}\right)+\sum_{i=1}^{N} \sum_{j \in G_{i}} \nabla V_{i j} \dot{x}_{i}^{T} \\
& =\sum_{i=1}^{N} \dot{x}_{i}^{T}\left(\ddot{x}_{i}+\sum_{j \in G_{i}} \nabla V_{i j}\right) .
\end{aligned}
$$

Combining (9) and (3), we get

$$
\begin{aligned}
\dot{\Phi} & =\sum_{i=1}^{N} \dot{x}_{i}^{T}\left(\ddot{x}_{i}+\sum_{j \in G_{i}} \nabla V_{i j}\right) \\
& =\sum_{i=1}^{N} \dot{x}_{i}^{T}\left(\sum_{j \in G_{i}} F_{i j}-k_{d} \dot{x}_{i}+\sum_{j \in G_{i}} \nabla V_{i j}\right) \\
& =\sum_{i=1}^{N} \dot{x}_{i}^{T}\left(\sum_{j \in G_{i}}-\nabla V_{i j}-k_{d} \dot{x}_{i}+\sum_{j \in G_{i}} \nabla V_{i j}\right) \\
& =-k_{d} \sum_{i=1}^{N} \dot{x}_{i}^{T} \dot{x}_{i},
\end{aligned}
$$

where $k_{d}$ is the damping coefficient and $\dot{\Phi}$ is seminegative definite. According to Lyapunov stability theory, the redeployment algorithm is asymptotically stable.

4.2. Redeployment Algorithm for Heterogeneous WSNs. As exponential force model can achieve fast convergence, the force model is shown in (11) and Figure 1:

$$
F_{i j}(d)= \begin{cases}\boldsymbol{\alpha}\left(d_{i j}^{-\beta}-D_{t h}^{-\beta}\right) \mathbf{u}_{i j}, & \text { if } D_{t h}<d_{i j}<R_{\mathrm{c}} \\ 0, & \text { if } d_{i j}=D_{t h} \\ \left|\boldsymbol{\alpha}\left(d_{i j}^{-\beta}-D_{t h}^{-\beta}\right)\right| \mathbf{u}_{i j}, & \text { if } 0.5 D_{t h}<d_{i j}<D_{t h} \\ 1 \mathbf{u}_{i j} & \text { if } d_{i j} \leq 0.5 D_{t h},\end{cases}
$$

where $\mathbf{u}_{i j}$ is the unit vector from node $i$ to node $j, \boldsymbol{\alpha}$ and $\beta$ are constants that may be changed in different situations, and $D_{t h}$ is the ideal distance.

In heterogeneous WSNs there is no ideal deployment to achieve, which is different from homogeneous WSNs, so a new balance point $D_{t h(i, j)}$ and the new force field truncation coefficient which keeps the nodes from stacking should be 


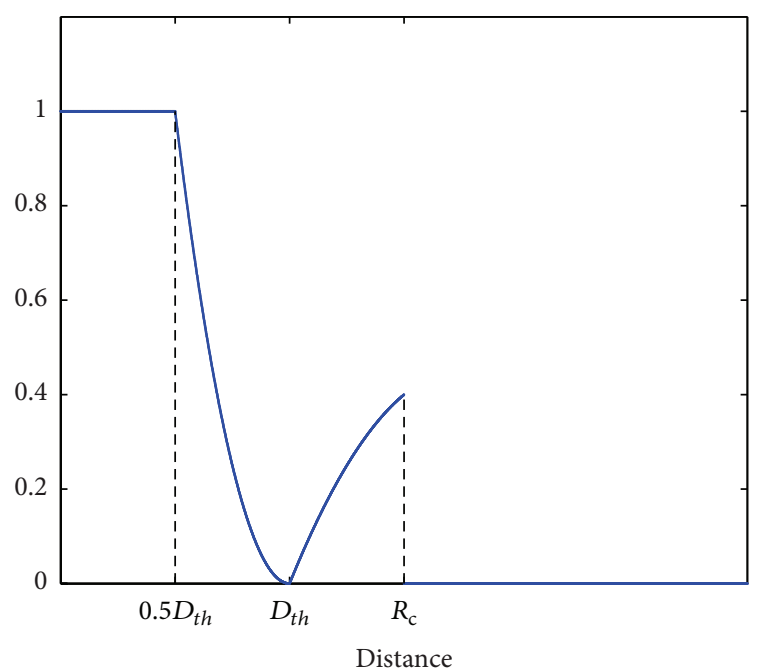

FIGURE 1: Virtual force model.

found. $D_{t h(i, j)}$ in homogeneous WSNs is $\sqrt{3} R_{s}$. However, in heterogeneous WSNs every node has different $R_{\mathrm{s}}$, so a new ideal distance for heterogeneous WSNs should be found.

Theorem 1. As shown in Figure 2, there are three nodes $i, j$, and $k$ with different sensing range.

If $\sum \phi_{i j k}=\phi_{i j}+\phi_{i k}+\phi_{j k}>2 \pi$, there is a hole in coverage area; if $\sum \phi_{i j k}=\phi_{i j}+\phi_{i k}+\phi_{j k}<2 \pi$, redundant coverage exists in area; if $\sum \phi_{i j k}=\phi_{i j}+\phi_{i k}+\phi_{j k}=2 \pi$, neither coverage hole nor redundant coverage exists.

Assume the distance between node $i$ and node $j$ is $d_{i j}=$ $\lambda\left(R_{\mathrm{s} i}+R_{\mathrm{s} j}\right)$, where $\lambda$ is the distance coefficient. Then, if $\lambda=$ $\sqrt{3} / 2$, nodes cover the area without any hole.

Proof. Consider

$$
\begin{aligned}
\phi_{i j} & =\arccos \left(\frac{R_{\mathrm{s} i}^{2}+R_{\mathrm{s} j}^{2}-d_{i j}^{2}}{2 R_{\mathrm{s} i} R_{\mathrm{s} j}}\right) \\
& =\arccos \left(\frac{R_{\mathrm{s} i}^{2}+R_{\mathrm{s} j}^{2}-0.75\left(R_{\mathrm{s} i}+R_{\mathrm{s} j}\right)^{2}}{2 R_{\mathrm{s} i} R_{\mathrm{s} j}}\right) \\
& =\arccos \left(\frac{0.25 R_{\mathrm{s} i}^{2}+0.25 R_{\mathrm{s} j}^{2}-1.5 R_{\mathrm{s} i} R_{\mathrm{s} j}}{2 R_{\mathrm{s} i} R_{\mathrm{s} j}}\right) \\
& =\arccos \left(\frac{0.25\left(R_{\mathrm{s} i}-R_{\mathrm{s} j}\right)^{2}}{2 R_{\mathrm{s} i} R_{\mathrm{s} j}}-0.5\right. \\
& \leq \arccos (-0.5)=\frac{2}{3} \pi .
\end{aligned}
$$
up.

Similarly $\phi_{i k} \leq(2 / 3) \pi$ and $\phi_{j k} \leq(2 / 3) \pi$, and sum them

Consider $\sum \phi_{i j k}=\phi_{i j}+\phi_{i k}+\phi_{j k} \leq 2 \pi$; that is, in heterogeneous sensor network, if we set $\lambda=\sqrt{3} / 2$, we can get coverage results without holes.

From the above, $D_{\operatorname{th}(i, j)}=(\sqrt{3} / 2)\left(R_{\mathrm{s} i}+R_{\mathrm{s} j}\right)$.
In VFA for homogeneous WSNs, there is a force field truncation coefficient $\lambda_{2}$; generally $\lambda_{2}=1$ which equivalent reduce the value of $R_{\mathrm{c}}$ in order to avoid nodes stacking when the nodes have high $R_{\mathrm{c}}$. However the connectivity of the networks cannot be ensured in the case of scatter initial deployment.

In this paper we employ Delaunay triangulation to solve the node gathering problem and on the other hand avoid affecting the connectivity of the networks.

Delaunay triangulation graph is composed of several disjoint triangles. The vertices of the triangle are sensor nodes. Delaunay figure is used as the logical topology of the network in the coverage control because it has no intersecting edge topology.

This paper uses Delaunay graph as the network topology where the balance point $D_{t h(i, j)}=\sqrt{3} / 2\left(R_{s i}+R_{\mathrm{s} j}\right)$.

To make the algorithm as a distributed algorithm, every node uses its own local Delaunay triangulation graph.

Definition 2. Every node and its neighbor nodes in its communication radius make up the Delaunay graph. This graph is the node's local Delaunay triangulation graph (Local DTG or LDTG). The LDTGs of all the nodes form Unit Delaunay Triangulation Graph (Unit DTG or UDTG). Obviously, UDTG is a subgraph of DTG.

To achieve better performance, another round of movement is employed; the proof is shown below.

Theorem 3. If $D_{t h(i, j)}=\sqrt{3} / 2\left(R_{\mathrm{s} i}+R_{\mathrm{s} j}\right)$ in a Delaunay triangle, the increment of $\angle j_{k} i j_{k+1}$ causes monotone decrement of $R_{\mathrm{s} i}$.

\section{Proof. Consider}

$$
\begin{aligned}
\cos \angle & j_{k} i j_{k+1} \\
= & \left(\left(\left(\frac{\sqrt{3}}{2}\right)\left(R_{\mathrm{s} i}+R_{\mathrm{s} j}\right)\right)^{2}\right. \\
& +\left(\left(\frac{\sqrt{3}}{2}\right)\left(R_{\mathrm{s} i}+R_{\mathrm{s}(j+1)}\right)\right)^{2} \\
& \left.-\left(\left(\frac{\sqrt{3}}{2}\right)\left(R_{\mathrm{s} j}+R_{\mathrm{s}(j+1)}\right)\right)^{2}\right) \\
& \times\left(2\left(\left(\frac{\sqrt{3}}{2}\right)\left(R_{\mathrm{s} i}+R_{\mathrm{s} j}\right)\left(\frac{\sqrt{3}}{2}\right)\left(R_{\mathrm{s} i}+R_{\mathrm{s}(j+1)}\right)\right)\right)^{-1} \\
= & \frac{\left(R_{\mathrm{s} i}+R_{\mathrm{s} j}\right)^{2}+\left(R_{\mathrm{s} i}+R_{\mathrm{s}(j+1)}\right)^{2}-\left(R_{\mathrm{s} j}+R_{\mathrm{s}(j+1)}\right)^{2}}{2\left(R_{\mathrm{s} i}+R_{\mathrm{s} j}\right)\left(R_{\mathrm{s} i}+R_{\mathrm{s}(j+1)}\right)} .
\end{aligned}
$$

Assuming three circles are tangent to each other, $\cos \angle j_{k} i j_{k+1}=\sqrt{3} / 2$ as shown in Figure 3 .

It is clear that

$$
\angle j_{k} i j_{k+1}=2 \arctan \left(\frac{R_{\mathrm{VL}}}{R_{\mathrm{si}}}\right) .
$$




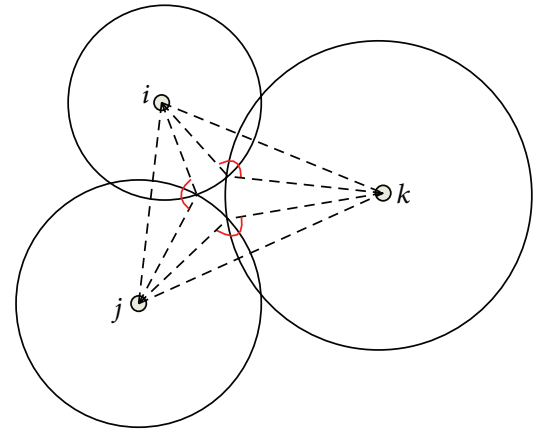

(a) $\sum \phi_{i j k}>2 \pi$

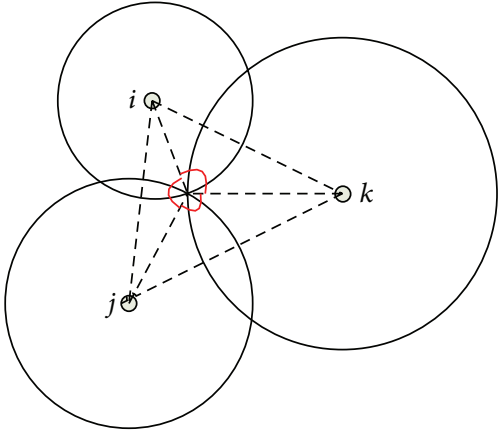

(b) $\sum \phi_{i j k}=2 \pi$

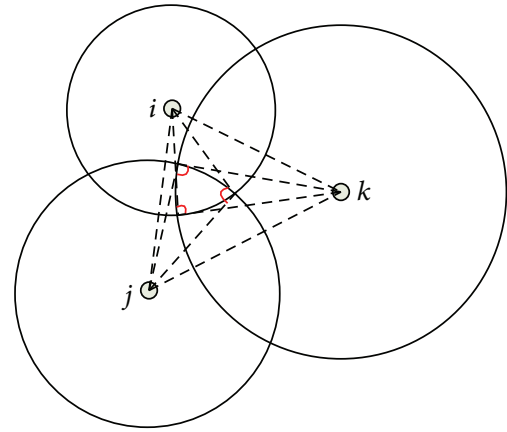

(c) $\sum \phi_{i j k}<2 \pi$

FIGURE 2: Localization effect in convex and concave holes.

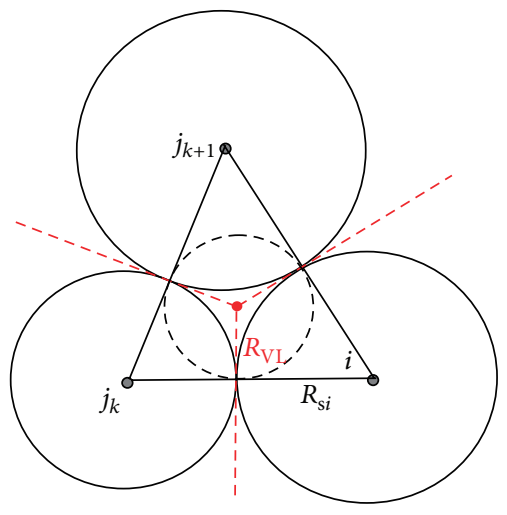

Figure 3: Delaunay triangle.

The area of the triangle can be described as follows:

$$
\begin{aligned}
S_{j_{k} i j_{k+1}} & =2 \times \frac{1}{2} R_{\mathrm{VL}} R_{\mathrm{s} i}+2 \times \frac{1}{2} R_{\mathrm{VL}} R_{\mathrm{s} j_{k}}+2 \times \frac{1}{2} R_{\mathrm{VL}} R_{\mathrm{s} j_{k+1}} \\
& =R_{\mathrm{VL}}\left(R_{\mathrm{si}}+R_{\mathrm{s} j_{k}}+R_{\mathrm{s} j_{k+1}}\right) .
\end{aligned}
$$

Also the area of the triangle can be calculated by Heron's formula:

$$
S_{j_{k} j_{k+1}}=\sqrt{\left(R_{s i}+R_{s j_{k}}+R_{s j_{k+1}}\right)\left(R_{s i} R_{s j_{k}} R_{s j_{k+1}}\right)} .
$$

Clearly from (14), (15), and (16),

$$
\angle j_{k} i j_{k+1}=2 \arctan \left(\sqrt{\frac{R_{s j_{k}} R_{s j_{k+1}}}{R_{s i}\left(R_{s i}+R_{s j_{k}}+R_{s j_{k+1}}\right)}}\right) .
$$

$\partial \angle j_{k} i j_{k+1} / \partial R_{s i}$ is shown in

$$
\begin{aligned}
\frac{\partial L j_{k} i j_{k+1}}{\partial R_{s i}}= & \frac{1}{1+R_{s j_{k}} R_{s j_{k+1}} / R_{s i}\left(R_{s i}+R_{s j_{k}}+R_{s j_{k+1}}\right)} \\
& \times \frac{1}{\sqrt{R_{s j_{k}} R_{s j_{k+1}} / R_{s i}\left(R_{s i}+R_{s j_{k}}+R_{s j_{k+1}}\right)}} \\
& \times\left(-\frac{R_{s j_{k}} R_{s j_{k+1}}}{R_{s i}^{2}\left(R_{s i}+R_{s j_{k}}+R_{s j_{k+1}}\right)}\right. \\
& \left.\quad-\frac{R_{s j_{k}} R_{s j_{k+1}}}{R_{s i}\left(R_{s i}+R_{s j_{k}}+R_{s j_{k+1}}\right)^{2}}\right)<0 .
\end{aligned}
$$

Theorem 3 proves that the increment of $\angle j_{k} i j_{k+1}$ leads to the monotone decrement of $R_{s i}$ in Delaunay triangle. Therefore a node in each Delaunay triangle that meets the distance coefficient must be a determined value. But it is almost impossible to have all nodes meet the value at one time. So another step is used to improve the performance.

As shown in Figure 2, if $\sum \phi_{i j k}=\phi_{i j}+\phi_{i k}+\phi_{j k}=2 \pi$, neither coverage holes nor coverage redundant exist. Then the main aim of the algorithm is to adjust the distance between the nodes according to the $\sum \phi_{i j k}$ in Delaunay triangle to approach $2 \pi$.

For node $i$ shown in Figure 2(a),

$$
\phi_{t h\left(i, j_{k}\right)}=\phi_{i j_{k}}+\left(2 \pi-\sum \phi_{i j_{k} j_{k+1}}\right) \frac{\phi_{i j_{k}}}{\sum \phi_{i j_{k} j_{k+1}}} .
$$
follows:

The ideal distance between nodes can be described as

$$
D_{t h\left(i, j_{k}\right)}=\left(R_{s i}^{2}+R_{s j_{k}}^{2}-2 R_{s i} R_{s j_{k}} \cos \phi_{t h\left(i, j_{k}\right)}\right) .
$$


$/ / m$ : the number of the logic neighbors of node $i$

// $\Delta t$ : the sampling time

Step 1. Find out logic neighbors node $i$ in $\operatorname{LDTG}(i)$ of node $i$;

Step 2. For $1 \leq j \leq m$

Step 2.1. Set distance coefficient $\lambda=\sqrt{3} / 2$

Step 2.2.

Calculate $\mathbf{F}_{i j}$ the virtual force between node $i$ and its neighbor node $j$;

Step 3. Calculate the resultant virtual force;

Step 4. Calculate the accelerated speed $a_{i}$ and speed $v_{i}$ of node $I$ and then move node $i$ to new position;

Step 5. Update $t+\Delta t$;

Step 6. Go back to Step 1.

Step 7. Find out logic neighbors node $i$ in $\operatorname{LDTG}(i)$ of node $i$;

Step 8. For $1 \leq j \leq m$

Step 8.1. Calculate the virtual force $\mathbf{F}_{i j}$ between node $i$ and its logic neighbor $j$;

Step 9. Calculate the resultant virtual force;

Step 10. Calculate the accelerated speed $a_{i}$ of node $i$ and speed $v_{i}$, node $i$ move to new position;

Step 11. Update to $t+\Delta t$

Step 12. Go back to Step 1.

Algorithm 1

Virtual force between nodes $i$ and $j$ is listed as follows:

$$
\begin{aligned}
& F_{i j}(d) \\
& = \begin{cases}\boldsymbol{\alpha}\left(d_{i j}^{-\beta}-D_{t h}^{-\beta}\right) \mathbf{u}_{i j}, & \text { if } D_{t h(i, j)}<d_{i j}<R_{c} \\
0, & \text { if } d_{i j}=D_{t h(i, j)} \text { or } E(i, j) \\
\left|\boldsymbol{\alpha}\left(d_{i j}^{-\beta}-D_{t h}^{-\beta}\right)\right| \mathbf{u}_{i j}, & \text { if } 0.5 D_{t h(i, j)}<d_{i j}<D_{t h(i, j)} \\
1 \mathbf{u}_{i j} & \text { if } d_{i j} \leq 0.5 D_{t h(i, j)} .\end{cases}
\end{aligned}
$$

If $E(i, j)$ is the outside edge of the LDTG, the virtual force between nodes $i$ and $j$ is zero.

Then each node calculates its own resultant virtual force of its logic neighbors. Then it moves to the new position according to the motion dynamic model.

Furthermore, the algorithm above can be described in Algorithm 1.

4.3. Energy Balanced Revision. Actually the imbalanced energy cost of the network may cause some node to run out of energy in a short time. In this case the whole structure of the WSNs may change, the QoS of the network cannot be guaranteed, and the lifetime of the network may be greatly shortened. As analyzed above, in mobile WSNs we can prolong the lifetime of the whole network by balancing the energy cost of the nodes during the redeployment process. Minimum energy of node $\bar{E}_{\text {min }}$ is to describe the minimum remaining energy of the nodes in the network.

Definition 4. $\overline{L_{x}}, \overline{L_{-x}}, \overline{L_{y}}$, and $\overline{L_{-y}}$ are the energy cost of the node when moving standard distance in each direction. Energy cost of node $i$ is shown as follows:

$$
\overline{L_{i}}=\sum_{\forall \operatorname{dir}}\left|\overline{L_{i}} \times \overline{\operatorname{dir}_{i}}\right|
$$

where $\overline{\operatorname{dir}_{i}}$ is the distance that node $i$ moves in each direction. $\overline{\mathrm{EI}_{i}}$ is the initial energy of node $i . \overline{E_{i}}$ is the energy of the node $i$. Thus the lowest energy of the node $\bar{E}_{\min }$ is described as follows:

$$
\bar{E}_{\min }=\operatorname{Min}\left(\overline{\mathrm{EI}_{i}}-\overline{L_{i}}\right) .
$$

In order to prolong the lifetime of the network, the algorithm should keep $\bar{E}_{\text {min }}$ as large as possible.

Definition 5. Energy differential coefficient $\varphi$ (see (24)) is used to describe the energy difference between two nodes:

$$
\boldsymbol{\varphi}_{i, j}=\frac{\left|\overline{E_{i}}-\overline{E_{j}}\right|}{\operatorname{Max}\left(\overline{E_{i}}, \overline{E_{j}}\right)} .
$$

Obviously, $\boldsymbol{\varphi} \in[0,1]$, and if $\varphi$ approaches zero, more effort should be made to protect the nodes which have low energy to save their energy.

Definition 6. Node level $\gamma$ shows the importance of the node. In practical application some key nodes are more important than other nodes. Those key nodes can influence the QoS of the whole network. Therefore the algorithm should give priority to economize the energy of those key nodes.

To balance the energy cost of the node energy control function (see (25)) is added to (21):

$$
e\left(\varphi_{i, j}\right)= \begin{cases}\varepsilon & \left(\operatorname{rand}[0,1]<\varphi_{i, j}\right) \\ 1 & \left(\operatorname{rand}[0,1] \geq \varphi_{i, j}\right)\end{cases}
$$

$\varepsilon$ is the attenuation coefficient. Every time node $i$ calculates the virtual force of its logic neighbor $j$, node $i$ compares its energy with $j$ and gets the energy differential coefficient $\varphi_{i, j}$, and then node $i$ generates a random number $k \in[0,1]$. If $k<\varphi_{i, j}$, the virtual force would be limited so that 
$/ / m$ : the number of the logic neighbors of node $i$

// $\Delta t$ : the sampling time

Step 1. Find out logic neighbors node $i$ in $\operatorname{LDTG}(i)$ of node $i$;

Step 2. For $1 \leq j \leq m$

Step 2.1. Set distance coefficient $\lambda=\sqrt{3} / 2$

Step 2.2.

Calculate $\mathbf{F}_{i j}$ the virtual force between node $i$ and its neighbor node $j$;

Step 2.3.

Compare the energy of node $i$ and node $j$,

Step 2.4.

Calculate the energy differential coefficient $\varphi$ to fix the Virtual force,

Step 3. Calculate the resultant virtual force;

Step 4. Calculate the accelerated speed $a_{i}$ and speed $v_{i}$ of node $I$ and then move node $i$ to new position;

Step 5. Update $t+\Delta t$;

Step 6. Go back to Step 1 .

Step 7. Find out logic neighbors node $i$ in $\operatorname{LDTG}(i)$ of node $i$;

Step 8 . For $1 \leq j \leq m$

Step 8.1. Calculate the virtual force $\mathbf{F}_{i j}$ between node $i$ and its logic neighbor $j$;

Step 9. Calculate the resultant virtual force considering node level $\gamma$;

Step 10. Calculate the accelerated speed $a_{i}$ of node $i$ and speed $v_{i}$, node $i$ move to new position;

Step 11. Update to $t+\Delta t$

Step 12. Go back to Step 1.

\section{Algorithm 2: EBRA.}

the movement of the node $i$ would be reduced and in that case node $i$ may save its energy in this round.

Then the new virtual force between node $i$ and node $j$ is listed as follows:

$$
\mathbf{F}_{\mathrm{eb}}(i)=\sum_{j \in \mathrm{LN}} e\left(\varphi_{i, j}\right) \cdot F_{i j}(d),
$$

where LN is the set of logic neighbors of node $i$.

There are some key nodes in the networks, those nodes should always save energy no matter their neighbor's energy is low or not.

Node level control function is addressed in

$$
\delta(\gamma)=(\gamma-\mu)^{-\sigma},
$$

where $\mu$ and $\sigma$ are constants which can be adjusted according to different situations. And the final resultant virtual force $\mathbf{F}_{i}$ is described in

$$
\mathbf{F}_{i}=\delta(\gamma) \sum_{j \in \mathrm{LN}} e\left(\varphi_{i, j}\right) \cdot F_{i j}(d) .
$$

Then each node calculates its own resultant virtual force of its logic neighbors according to the distance and energy and node level. Then it moves to the new position according to the motion dynamic model.

EBRA can be described in Algorithm 2.

\subsection{Performance Evaluation}

Coverage Rate. The coverage rate is a measure of the coverage quality for WSNs. It was originally introduced by Gage [11]. In the blanket coverage problem, coverage is defined by the ratio of the union of covered areas of each node to the complete

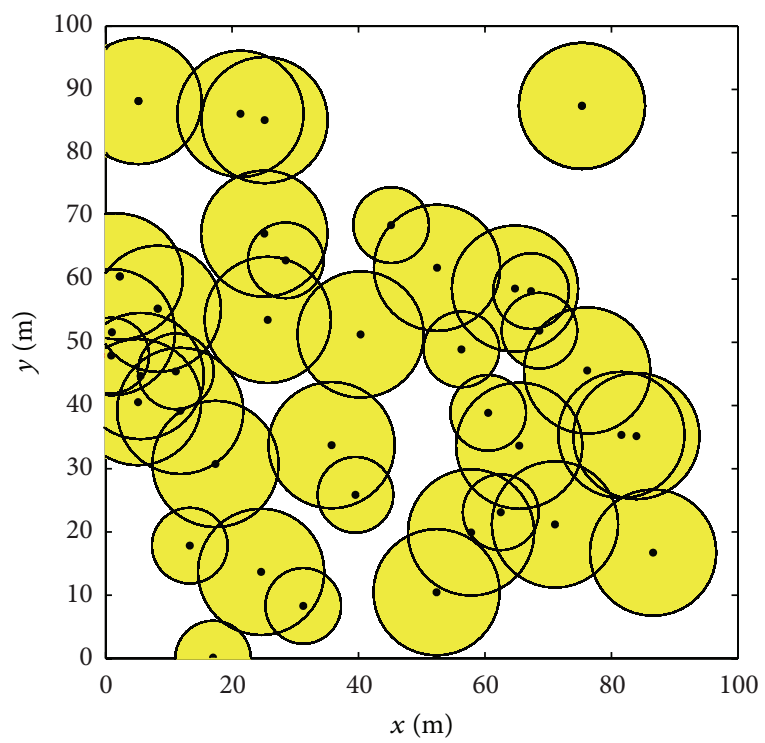

Figure 4: Scatter initial deployment.

area of interest. In this paper, the covered area of each node is defined as the circular area with sensing range $R_{s}$. The value of coverage rate $C$ is as follows:

$$
C=\frac{\bigcup_{i=1}^{n} A_{i}}{A},
$$

where $A_{i}$ is the area covered by the node $i, n$ is the total number of mobile sensor nodes, and $A$ is the total area. 


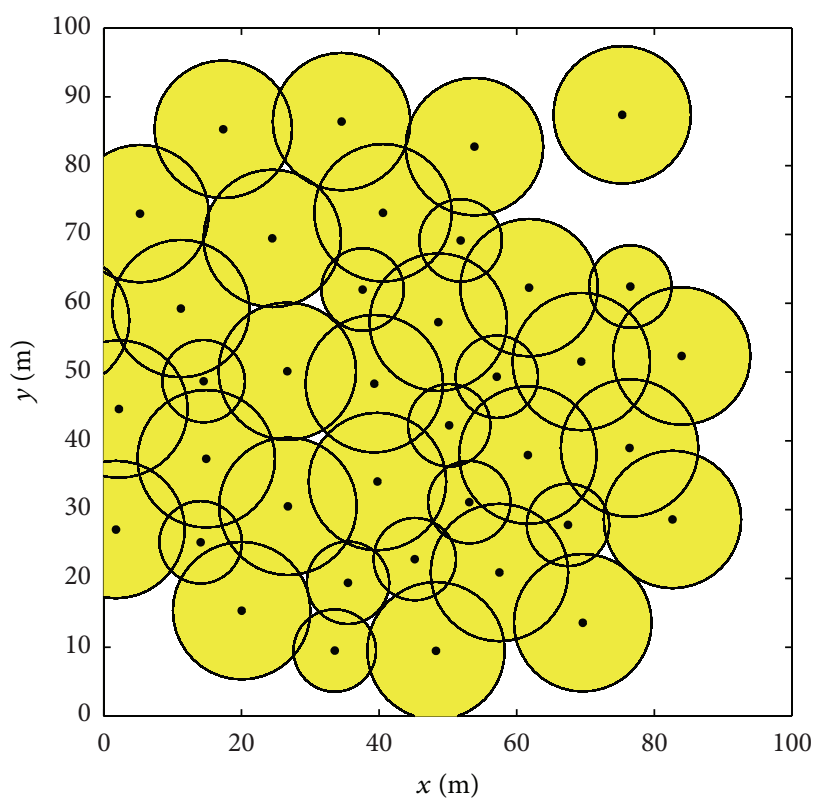

(a) Redeployment by VFA

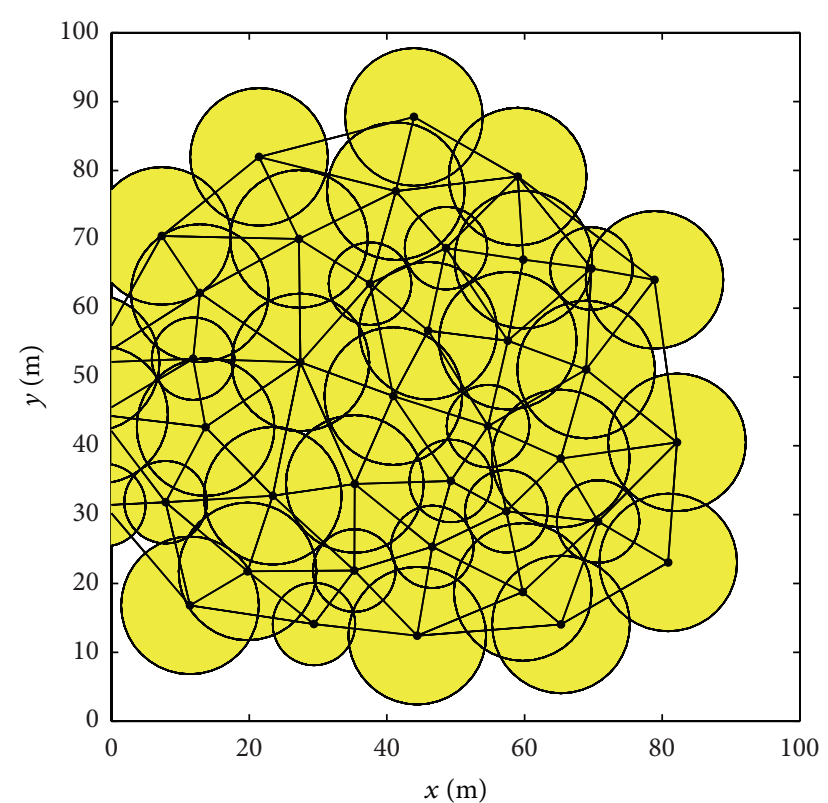

(b) Redeployment by EBRA

FIgURE 5: Simulation result in scatter initial deployment.

Uniformity of Energy. In heterogeneous WSNs, the uniformity of energy can be measured by the average of the local standard deviation of the node energy

$$
\sigma_{E}=\left(\frac{1}{n} \sum_{i=1}^{n}\left(E_{i}-\frac{1}{n} \sum_{j=1}^{n} E_{i}\right)^{2}\right)^{1 / 2} .
$$

Minimum Energy. $\bar{E}_{\text {min }}$ stands for the minimum energy of all the nodes and is described in (23).

\section{Numerical Simulation}

In this section, we present the simulation results of EBRA.

In the simulation environment, 40 nodes are deployed randomly in a $100 \mathrm{~m} * 100 \mathrm{~m}$ square field. Communication range of all nodes is $30 \mathrm{~m}$. The sensing range of 14 nodes is $10 \mathrm{~m}$, and other nodes' sensing range is $8 \mathrm{~m}$.

\subsection{Performance of Energy Balanced Redeployment Algorithm.} Firstly, simulation is done in scatter initial deployment. The scatter initial deployment is shown in Figure 4.

The redeployment results of VFA and EBRA in scatter initial deployment are shown in Figures 5(a) and 5(b).

It can be seen clearly from Figure 5 that EBRA shows the better result than VFA for guaranteeing the connectivity of the whole network. VFA employed force field truncation to avoid nodes stacking. $\lambda_{2}=1$ makes long-distance virtual force disappear. In EBRA use Delaunay triangulation to seek logic neighbor of nodes instead of real neighbor to settle the defects of VFA.

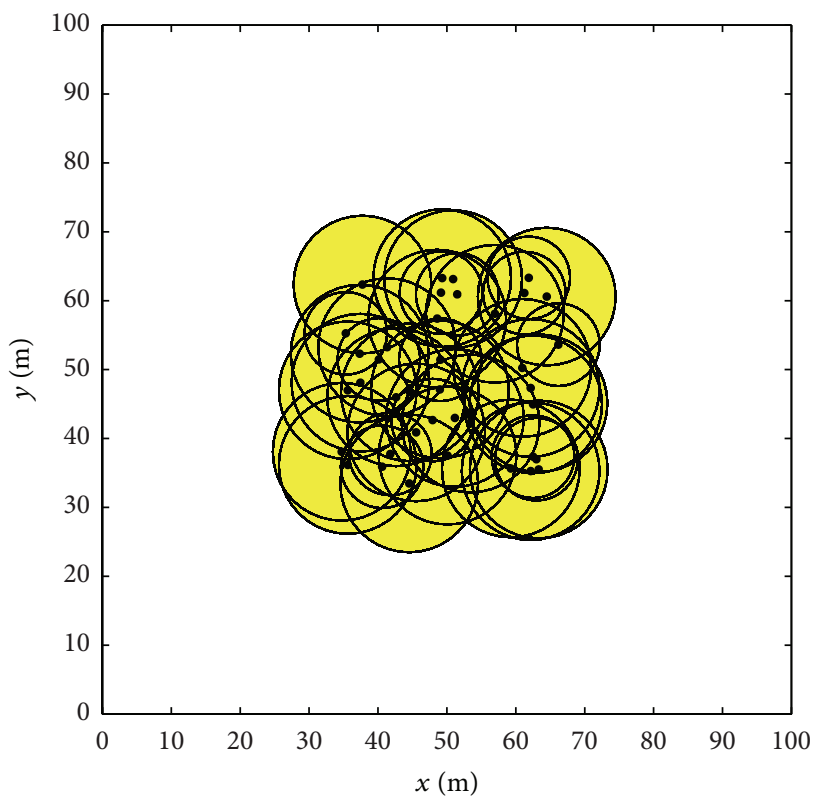

FIgure 6: Concentrated initial deployment.

Then simulation is done in concentrated initial deployment. The concentrated initial deployment is shown in Figure 6.

The redeployment results of VFA and EBRA in concentrated initial deployment are shown in Figures 7(a) and 7(b).

It can be seen clearly from Figure $7(\mathrm{a})$. Compared with Figure 7(b), less holes and redundant coverage exist in the deployment result. In VFA, ideal distance coefficient is settled, and neighbor nodes cannot reach the ideal distance 


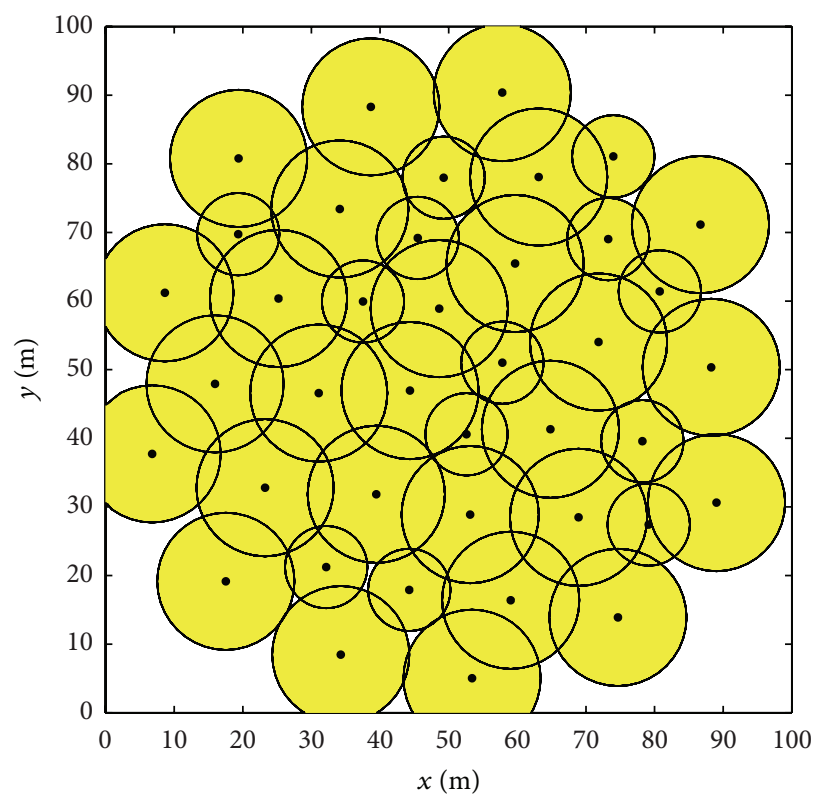

(a) Redeployment by VFA

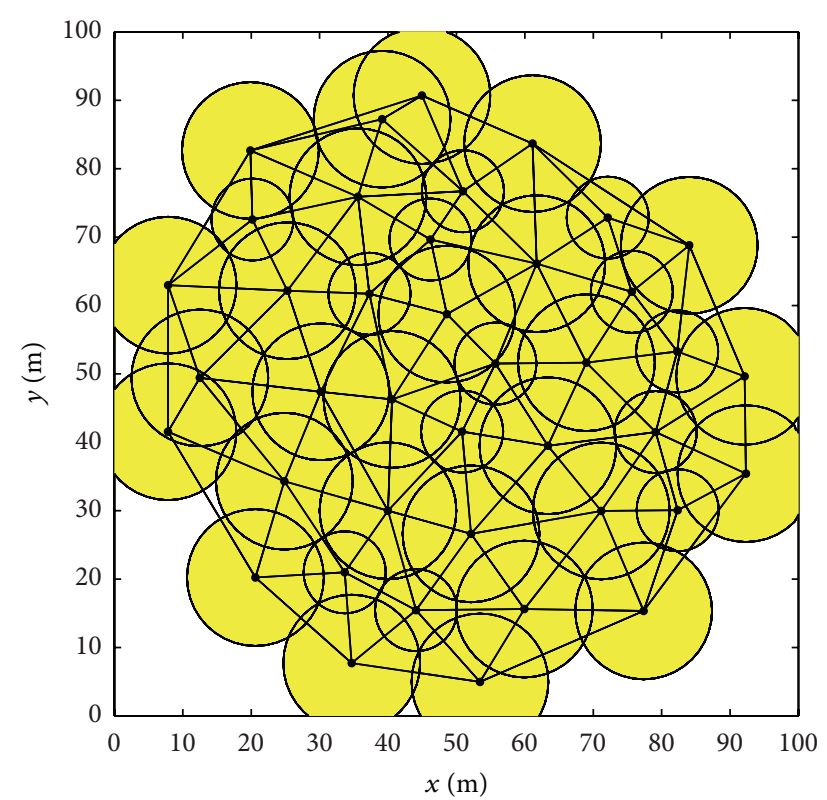

(b) Redeployment by EBRA

Figure 7: Simulation result in concentrated initial deployment.

coefficient at same time. To improve the performance of the algorithm, another round of movement begins with modified ideal distance coefficient. Due to the modified ideal distance coefficient, the virtual forces between nodes change causing two scenarios. In area with redundant coverage virtual force makes the nodes move away from each other and in area with holes virtual force makes nodes get closer to each other.

The above experiments have been carried out for 100 times, and the simulation results are illustrated below.

Figure 8 shows the $k$-coverage rates of VFA and EBRA with the initial deployment as shown in Figure 6.

It can be seen clearly that EBRA can achieve better deployment than VFA, because it has less redundant coverage, and holes.

Figure 9 shows the minimum energy of all the nodes in mobile WSNs. Compared with traditional VFA, the minimum energy value of nodes reduces more slowly, and at step 100, both algorithms meet the ending condition, and EBRA saves more energy for the mobile WSNs which means the mobile WSNs employed EBRA can provide longer service time and better QoS.

Figure 10 shows the uniformity of energy of the whole networks. The uniformity of energy of EBRA is lower than traditional VFA which means the energy in EBRA is more uniform distribution which provides better robustness for the whole system.

Figure 11 shows the energy cost of the key nodes with and without energy protecting. Simulations are done in same initial deployment. Firstly a random node is selected as a key node whose energy cost will be recoded. Secondly, the node which has been selected changes its node level and then recodes the energy cost. It can be seen clearly from Figure 11 that EBRA saves the energy for the key nodes.

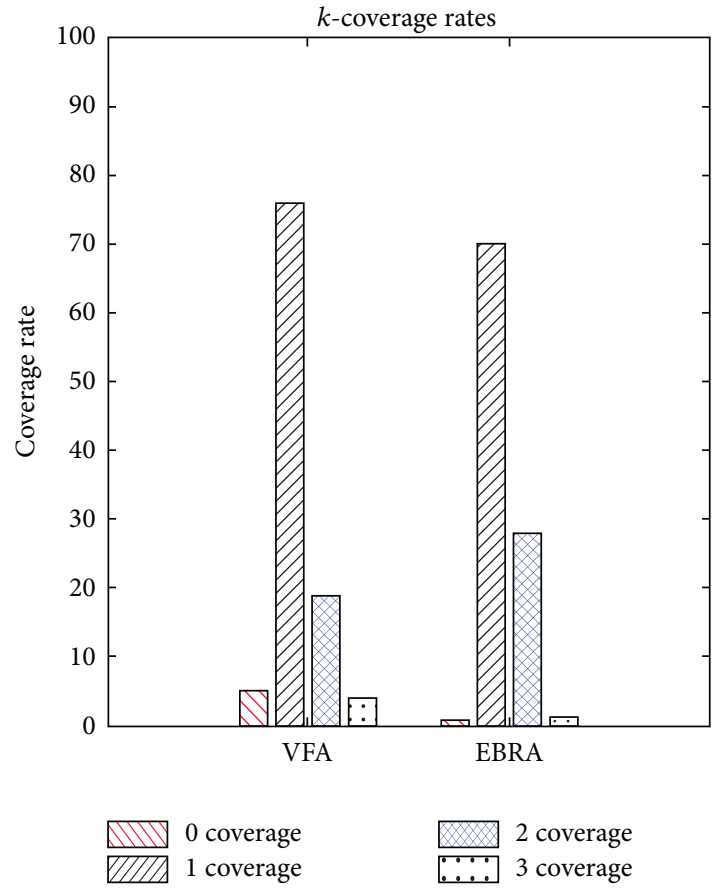

FIGURE $8: k$-coverage rates of the final deployment.

\section{Conclusion}

In this paper, we analyze the coverage problem in heterogeneous WSNs. We argue the energy unbalanced, coverage holes, and redundant coverage problems that appear when traditional VFA algorithms are used in heterogeneous WSNs. 


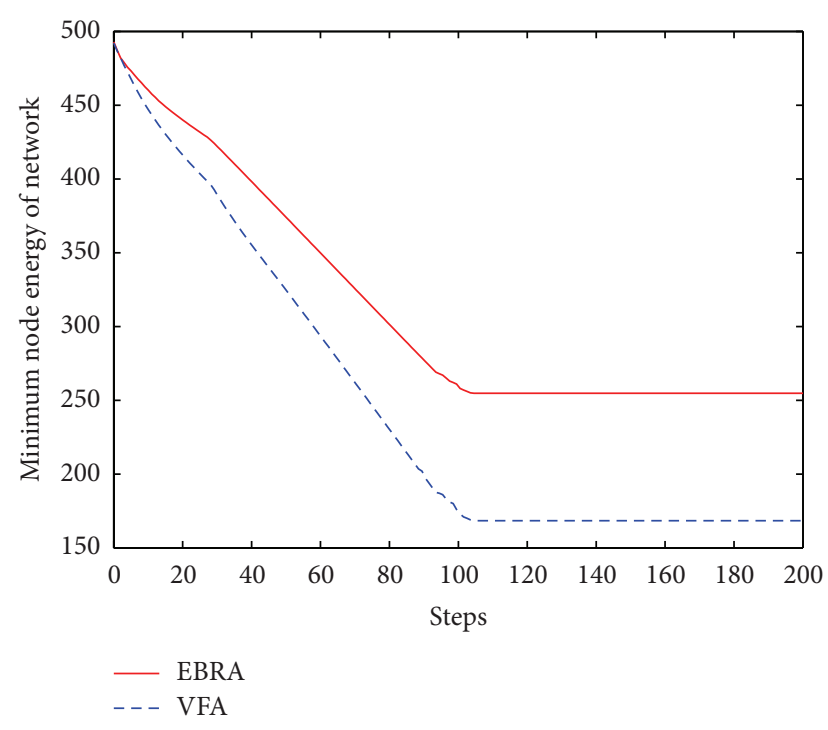

Figure 9: Minimum energy of node.

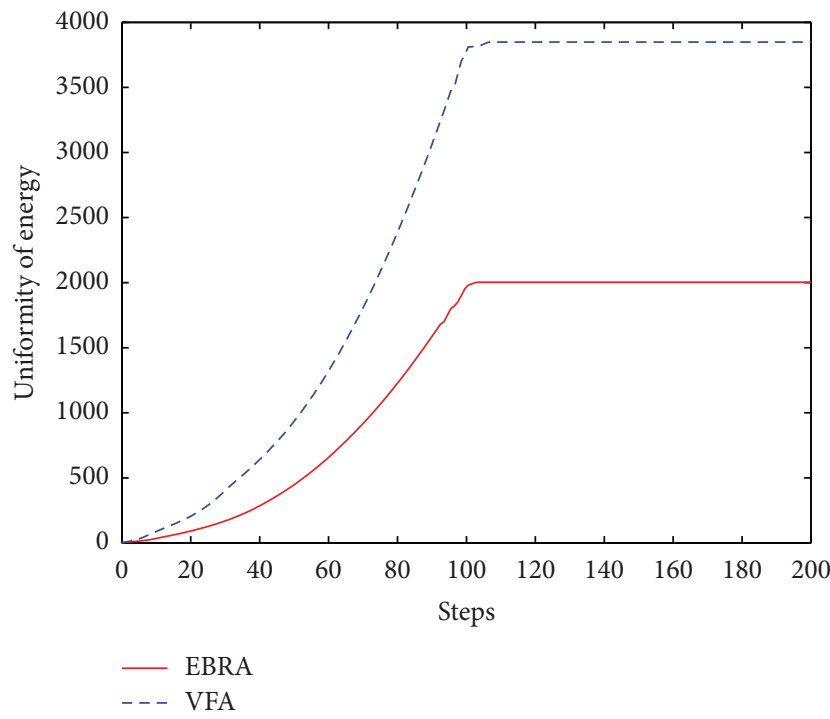

FIGURE 10: Uniformity of energy.

In order to solve these problems, EBRA which can be applied for heterogeneous WSNs in any initial deployment is proposed. Furthermore, it provides better performance than VFA. Moreover the energy cost of the nodes in the network has been balanced to prolong the lifetime and enhance the QoS of the WSNs (heterogeneous WSNs).

Compared with VFA, EBRA saves almost about 20\% energy for key nodes and reduces the energy waste of network. Moreover EBRA provides better coverage performance (larger coverage area, less redundant, and holes). All the above insure that EBRA is more suitable in redeployment heterogeneous WSNs for its longer lifetime, better coverage performance, and higher QoS. Future work involves studying $k$-coverage requirement of heterogeneous WSNs.

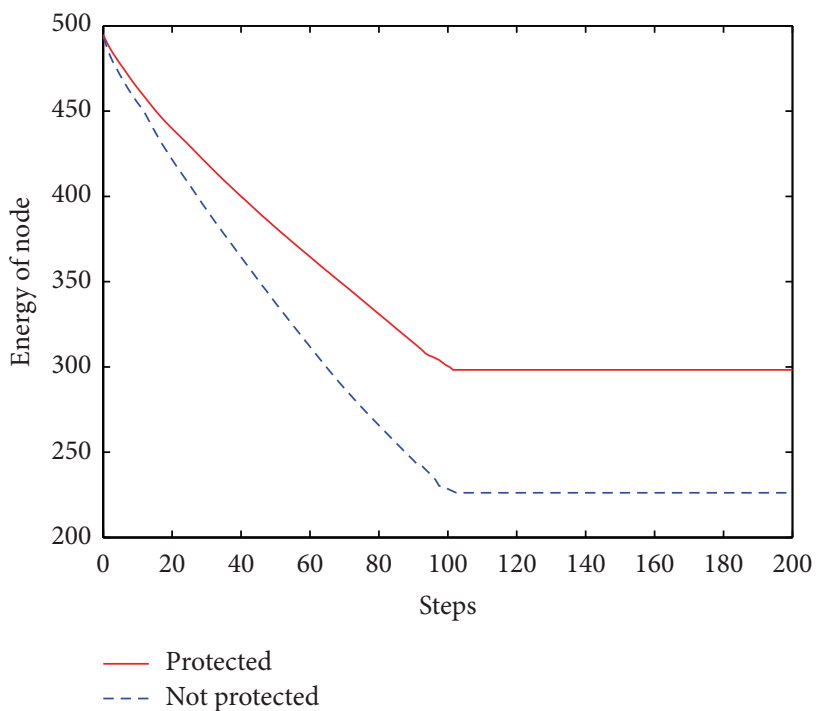

FIGURE 11: Energy cost of the key nodes with and without energy protecting.

\section{Conflict of Interests}

The authors declare that there is no conflict of interests regarding the publication of this paper.

\section{Acknowledgments}

This work was supported by National Defense Advanced Research Project of China (Grants nos. 104080 and 40405020401), National Natural Science Foundation of Youth Science Fund (no. 61203144 and no. 61104086), and Research Fund for the Doctoral Program of Higher Education of China (no. 20121101110032).

\section{References}

[1] L. Krishnamurthy, R. Adler, P. Buonadonna, J. Chhabra, M. Flanigan, and N. Kushalnagar, "Design and deployment of industrial sensor networks: experiences from a semiconductor plant and the North Sea," in Proceedings of the 3rd ACM International Conference on Embedded Networked Sensor Systems (SenSys '05), pp. 64-75, Association for Computing Machinery, San Diego, Calif, USA, November 2005.

[2] S. Meguerdichian, F. Koushanfar, M. Potkonjak, and M. B. Srivastava, "Coverage problems in wireless ad-hoc sensor networks," in Proceedings of the 20th Annual Joint Conference of the IEEE Computer and Communications Societies, pp. 1380-1387, IEEE, Piscataway, NJ, USA, April 2001.

[3] K. Derr and M. Manic, "Extended virtual spring mesh (EVSM): the distributed self-organizing mobile ad hoc network for area exploration," IEEE Transactions on Industrial Electronics, vol. 58, no. 12, pp. 5424-5437, 2011.

[4] R. S. Chang and S. H. Wang, "Self-deployment by density control in sensor networks," IEEE Transactions on Vehicular Technology, vol. 57, no. 3, pp. 1745-1755, 2008.

[5] E. J. Duarte-Melo and M. Liu, "Analysis of energy consumption and lifetime of heterogeneous wireless sensor networks," in 
Proceedings of the IEEE Global Telecommunications Conference (GLOBECOM '02), pp. 21-25, Institute of Electrical and Electronics Engineers, Taipei, Taiwan, November 2002.

[6] N. Heo and P. K. Varshney, "Energy-efficient deployment of intelligent mobile sensor networks," IEEE Transactions on Systems, Man, and Cybernetics Part A:Systems and Humans, vol. 35, no. 1, pp. 78-92, 2005.

[7] M.-L. Lam and Y.-H. Liu, "ISOGRID: an efficient algorithm for coverage enhancement in mobile sensor networks," in Proceedings of the IEEE/RSJ International Conference on Intelligent Robots and Systems (IROS '06), pp. 1458-1463, Piscataway, NJ, USA, October 2006.

[8] G. Wang, G. Cao, and T. L. Porta, "Movement-assisted sensor deployment," in Proceedings of the 23rd Annual Joint Conference of the IEEE Computer and Communications Societies (INFOCOM '04), vol. 4, pp. 2469-2479, March 2004.

[9] C. H. Wu, K. C. Lee, and Y. C. Chung, "A Delaunay Triangulation based method for wireless sensor network deployment," Computer Communications, vol. 30, no. 14-15, pp. 2744-2752, 2007.

[10] W. Li, Y. I. Kamil, and A. Manikas, "Wireless array based sensor relocation in mobile sensor networks," in Proceedings of the ACM International Wireless Communications and Mobile Computing Conference (IWCMC '09), Association for Computing Machinery, pp. 832-838, Leipzig, Germany, June 2009.

[11] D. W. Gage, "Development and command-control tools for many-robot systems," in Microrobotics and Micromechanical Systems, vol. 25, pp. 121-129, Society of Photo-Optical Instrumentation Engineers, Philadelphia, Pa, USA, 1995.

[12] C. Nieto-Granda, J. G. Rogers, and H. I. Christensen, Coordination Strategies for Multi-Robot Exploration and Mapping, Sage, Thousand Oaks, Calif, USA, 4th edition, 2014.

[13] X. Wang and S. Wang, "Hierarchical deployment optimization for wireless sensor networks," IEEE Transactions on Mobile Computing, vol. 10, no. 7, pp. 1028-1041, 2011.

[14] Y. Zou and K. Chakrabarty, "Sensor deployment and target localization based on virtual forces," in Proceedings of the 22nd Annual Joint Conference of the IEEE Computer and Communications Societies (IEEE INFOCOM '03), pp. 1293-1303, Institute of Electrical and Electronics Engineers, San Francisco, Calif, USA, March 2003.

[15] H. Nojeong and P. K. Varshney, "A distributed self spreading algorithm for mobile wireless sensor networks," in Proceedings of the IEEE Wireless Communications and Networking Conference (WCNC '03), vol. 3, pp. 1597-1602, 2003. 


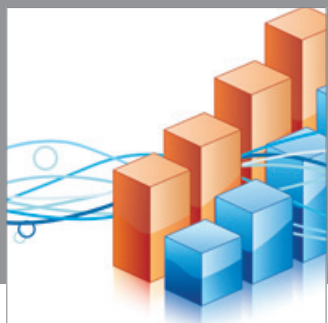

Advances in

Operations Research

mansans

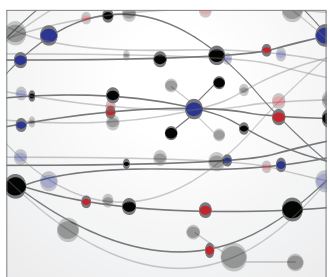

The Scientific World Journal
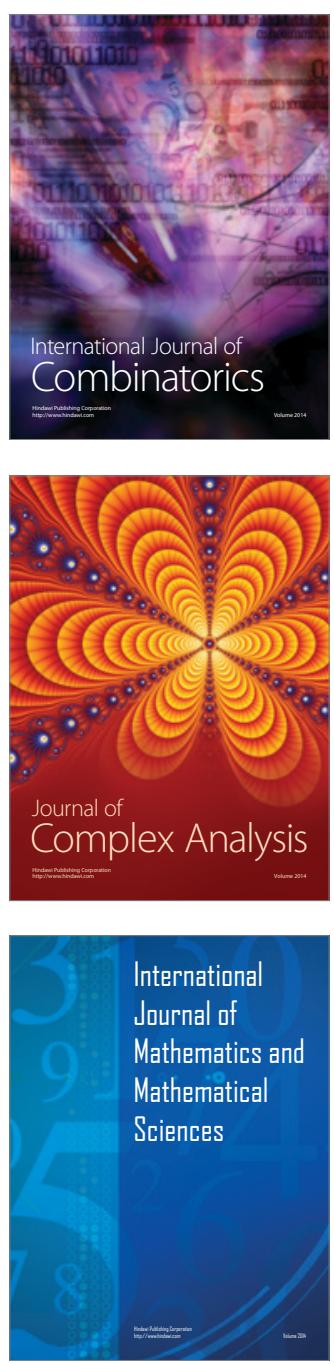
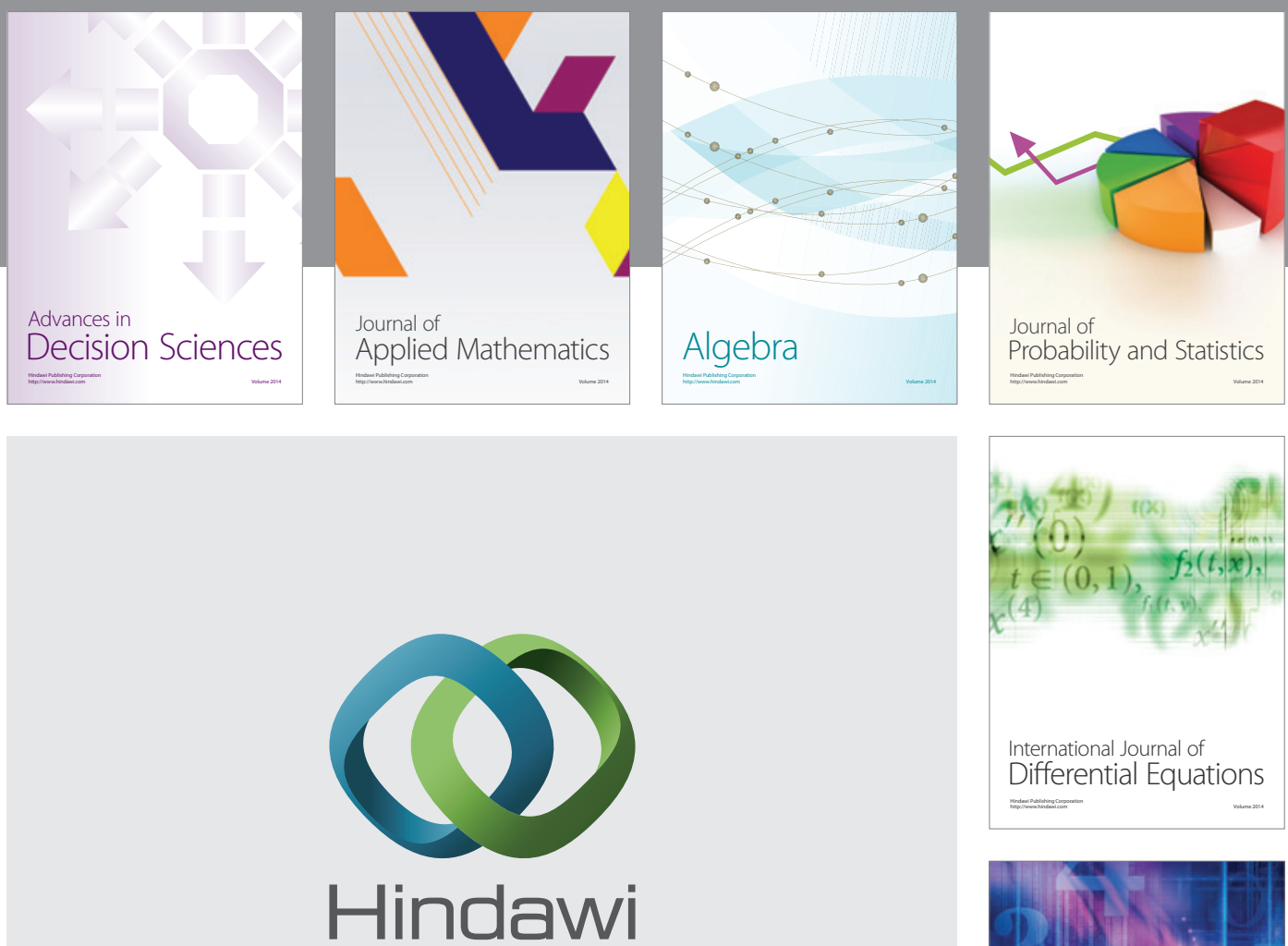

Submit your manuscripts at http://www.hindawi.com
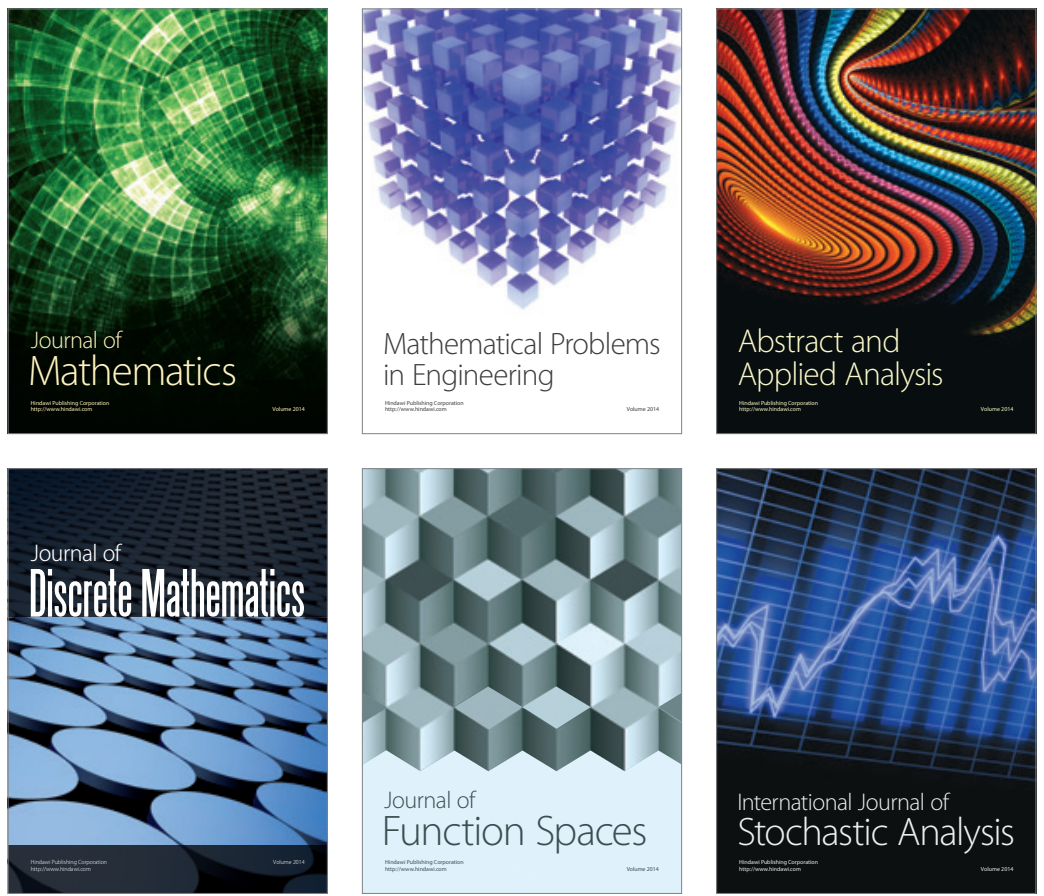

Journal of

Function Spaces

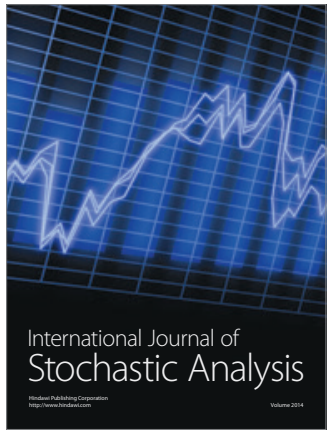

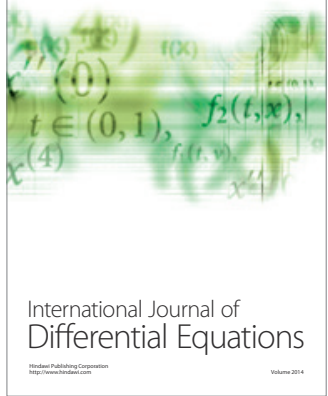
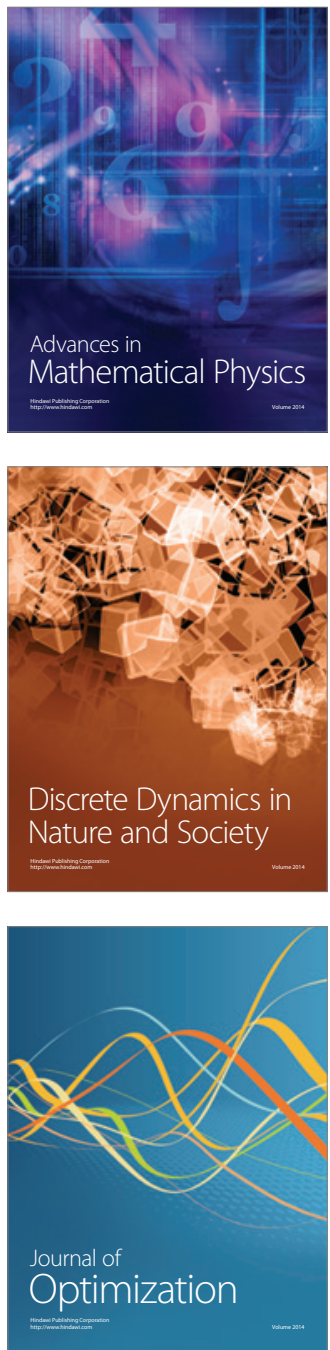